\title{
Signatures of Noncommutative Geometry in Muon Decay for Nonsymmetric Gravity
}

\author{
Dinesh Singh $*$ Nader Mobed $\mathbb{H}^{\dagger}$ and Pierre-Philippe Ouimet \\ Department of Physics, University of Regina, Regina, Saskatchewan, S4S 0A2, Canada
}

(Dated: October 31, 2018)

\begin{abstract}
It is shown how to identify potential signatures of noncommutative geometry within the decay spectrum of a muon in orbit near the event horizon of a microscopic Schwarzschild black hole. This possibility follows from a re-interpretation of J.W. Moffat's nonsymmetric theory of gravity, first published in Phys. Rev. D 19, 3554 (1979), where the antisymmetric part of the metric tensor manifests the hypothesized noncommutative geometric structure throughout the manifold. It is further shown that for a given sign convention, the predicted signatures counteract the effects of curvature-induced muon stabilization predicted by D. Singh and N. Mobed in Phys. Rev. D 79, 024026 (2009). While it is unclear whether evidence for noncommutative geometry may be found at the Large Hadron Collider (LHC) anytime soon, this approach at least provides a useful direction for future quantum gravity research based on the ideas presented here.
\end{abstract}

PACS numbers: 02.40.Gh, 04.60.Bc, 04.62.+v, 11.30.Cp, 13.35.Bv

Introduction.-Efforts to find a unified theory of particle physics beyond the Standard Model (SM) have led researchers to consider various approaches towards reaching this goal. One possibility that has gained widespread attention over the past two decades is string theory [1], primarily because of the claim that it includes a quantum theory of gravity within its framework. However, there are numerous competing formulations, such as loop quantum gravity [2] and others, with radically different foundations and implications as they relate to BeyondStandard-Model (BSM) physics. This is due to the fact that the SM offers no insights about how gravity behaves at quantum length scales.

A recent approach in quantum gravity of serious consideration is noncommutative geometry [3], which assumes that pairs of space-time co-ordinates violate commutation at some scale $k$ with dimensions of area, and instead behave like noncommutating operators. This property is described by

$$
\left[\boldsymbol{X}^{\mu}, \boldsymbol{X}^{\nu}\right]=i \hbar \boldsymbol{J}^{\mu \nu}\left(\boldsymbol{X}^{\alpha}\right)
$$

where $\boldsymbol{X}^{\mu}$ is a Hermitian co-ordinate operator and $\boldsymbol{J}^{\mu \nu}$ is a dimensionless antisymmetric Hermitian operator, resembling orbital angular momentum generators with an $\mathrm{SU}(2)$ Lie algebra structure. Historically, this proposal was introduced several decades ago [4] to truncate ultraviolet divergences in the early developments of QED, but lost favour after successful techniques of regularization and renormalization were introduced. Nonetheless, noncommutative geometry has made a resurgence of late, in part because of its capacity to offset curvature singularities inside black holes [5] by truncating the curvature field strength in much the same way as was done to overcome perceived deficiencies in quantum field theory.

In contrast to taking a direct approach for quantum gravity research, a recent investigation [6] focussed on the more intermediate problem of understanding the applicability of the Poincaré group, the space-time symmetry group for classifying elementary particles according to their mass and spin angular momentum, for situations involving the non-inertial motion of spin- $1 / 2$ particles in space with respect to a global laboratory frame in a flat space-time background. This is a relevant issue given that the Poincaré group is well-defined only for strictly inertial motion [6], leading to conceptual challenges when applied to an elementary particle's accelerated motion in flat space-time or geodesic motion in curved spacetime. By describing the Poincaré group generators for the canonical momentum $\boldsymbol{P}$ in terms of curvilinear spatial co-ordinates to accommodate the symmetries of the particle's classical spatial trajectory, it is shown that the corresponding Pauli-Lubanski spin four-vector $\boldsymbol{W}$ yields a Casimir scalar $\boldsymbol{W} \cdot \boldsymbol{W}$ that is no longer the Lorentz invariant $\frac{1}{2}\left(\frac{1}{2}+1\right) m_{0}^{2}$, where $m_{0}^{2}=\boldsymbol{P} \cdot \boldsymbol{P}$. Rather, $\boldsymbol{W} \cdot \boldsymbol{W}$ is now frame dependent due to an additive term coupling the Pauli spin operator $\boldsymbol{\sigma}$ with a Hermitian three-vector $\boldsymbol{R}^{i}=\left(\frac{i}{2 \hbar}\right) \epsilon^{i j k}\left[\boldsymbol{P}_{j}, \boldsymbol{P}_{k}\right]$, known as the non-inertial dipole operator [6, 7] because it generates an interaction term analogous to a dipole interaction with a magnetic field. For $r$ the particle trajectory's local radius of curvature with respect to the laboratory frame, the dimensionality of $\boldsymbol{R}$ is $|\boldsymbol{P}| / r$, with the property that $\boldsymbol{R} \rightarrow \mathbf{0}$ for fixed momentum as $r \rightarrow \infty$, while for Cartesian co-ordinates to represent strictly rectilinear motion, $\boldsymbol{R}=\mathbf{0}$ identically.

More recently, this computation was repeated in the presence of a curved space-time background, described in terms of Fermi normal co-ordinates. Upon applying the formalism to the specific example of muon decay while orbiting a microscopic Kerr black hole [8], it was shown that the muon decay spectrum becomes significantly distorted due to both non-inertial effects from $\boldsymbol{R}$ and curvatureinduced contributions, ultimately leading to a predicted stabilization of the muon when approaching an orbital radius close to the particle's Compton wavelength. This by itself is a highly significant result, since it suggests that the intersection between quantum mechanics and 
gravitation becomes relevant at a length scale over 20 orders of magnitude larger than the Planck scale for this application, and suggests non-trivial behaviour of elementary particles in curved space-time apparently unaccounted for by current explorations of quantum gravity research. It also corroborates an earlier claim [9] that predicts curvature-induced changes to the electric field at the Compton scale generated by a charged microscopic Kerr black hole with the same mass and spin as an electron. In addition, this paper noted an interesting observation that if the metric tensor in Fermi normal co-ordinates ${ }^{F} g_{\mu \nu}(X)$ were allowed to become nonsymmetric while simultaneously allowing for $X^{\mu} \rightarrow \boldsymbol{X}^{\mu}$ in accordance with (1), then it is theoretically possible to identify non-trivial signatures of noncommutative geometry in the muon decay spectrum. However, a preliminary computation with this extension yielded no contribution whatsoever due to noncommutative geometry, a highly surprising result with no obvious explanation forthcoming.

Nonetheless, since this first investigation into noncommutative geometry, it was soon realized that a more complete computation to yield a signature would require the full details of a suitably-defined nonsymmetric theory of gravity. It so happens that a theory of nonsymmetric gravity developed years ago by Moffat [10, 11], originally derived as a purely classical theory with a different motivation in mind, may satisfy this requirement if the antisymmetric part of the metric tensor is identified exclusively with the noncommutative geometric extension of space-time, as originally proposed [8]. The purpose of this paper is to present the outcome of this computation in a microscopic Schwarzschild space-time background using Moffat's nonsymmetric gravity theory to incorporate additional curvature terms coupled to $k$ that were previously not considered. As well, this paper compares two possible formulations of noncommutative geometry, as determined by the choice for $\boldsymbol{J}^{\mu \nu}\left(\boldsymbol{X}^{\alpha}\right)$ in (1). While $k$ may have theoretical physical constraints on its magnitude, in this paper $\hbar$ is treated as a free parameter to be determined by observation. Geometric units of $G=c=1$ are assumed throughout, where the curvature tensors presented here satisfy the conventions of MTW [12] but with -2 metric signature.

Formalism.-While details of the formalism employed here are given elsewhere [8], it is important to offer some relevant highlights to justify its later extension to include noncommutative geometry. Given Fermi normal co-ordinates $X^{\mu}=\left(T, X^{i}\right)$ defined in a local neighbourhood about a spin- $1 / 2$ particle's worldline, where $T$ is the proper time and $X^{i}$ is the local Cartesian spatial coordinate in the Fermi frame, the covariant Dirac equation with mass $m$ and $\partial_{\mu}=\partial / \partial X^{\mu}$ is

$$
\left[i \gamma^{\mu}(X)\left(\partial_{\mu}+i \Gamma_{\mu}(X)\right)-m / \hbar\right] \psi(X)=0
$$

The set of gamma matrices $\left\{\gamma^{\mu}(X)\right\}$ satisfy $\left\{\gamma^{\mu}(X), \gamma^{\nu}(X)\right\}=2 g_{F}^{\mu \nu}(X)$ and $\Gamma_{\mu}(X)$ is the spin connection. An orthonormal vierbein set $\left\{\bar{e}_{\hat{\alpha}}^{\mu}(X)\right\}$ and its inverse $\left\{\bar{e}^{\hat{\alpha}}{ }_{\mu}(X)\right\}$ can be obtained to define a local Lorentz frame, denoted by co-ordinates with hatted indices. The metric tensor is then described by ${ }^{F} g_{\mu \nu}(X)=\eta_{\hat{\alpha} \hat{\beta}} \bar{e}^{\hat{\alpha}}{ }_{\mu}(X) \bar{e}^{\hat{\beta}}{ }_{\nu}(X)$, such that

$$
\begin{aligned}
& { }^{F} g_{00}(X)=1-{ }^{F} R_{l 00 m}(T) X^{l} X^{m}+\cdots, \\
& { }^{F} g_{0 j}(X)=-\frac{2}{3}{ }^{F} R_{l 0 j m}(T) X^{l} X^{m}+\cdots, \\
& { }^{F} g_{i j}(X)=\eta_{i j}-\frac{1}{3}{ }^{F} R_{l i j m}(T) X^{l} X^{m}+\cdots,
\end{aligned}
$$

where $\eta_{\mu \nu}$ is the Minkowski metric and ${ }^{F} R_{\mu \alpha \beta \nu}(T)$ is the projection of the Riemann tensor for general relativity (GR) onto the Fermi frame.

A straightforward conversion of (2) to mutually orthogonal spatial curvilinear co-ordinates is then introduced, defined by $U^{\mu}=\left(T, u^{i}\right)$, where $X^{i}=X^{i}(u)$. Subsequent projection of (2) onto the local Lorentz frame leads to

$$
\left[\gamma^{\hat{\mu}}\left(\boldsymbol{P}_{\hat{\mu}}-\hbar \boldsymbol{\Gamma}_{\hat{\mu}}(U)\right)-m\right] \psi(U)=0,
$$

where

$$
i \boldsymbol{\Gamma}_{\hat{\mu}}=\overline{\boldsymbol{\Gamma}}_{\hat{\mu}}^{(\mathrm{S})}+\gamma^{\hat{l}} \gamma^{\hat{m}} \overline{\boldsymbol{\Gamma}}_{\hat{0}[\hat{l} \hat{m}]}^{(\mathrm{T})} \delta_{\hat{\mu}}^{\hat{0}}
$$

and

$$
\begin{aligned}
\overline{\boldsymbol{\Gamma}}_{\hat{0}}^{(\mathrm{S})}= & \frac{1}{12}{ }^{F} R^{m}{ }_{j m k, 0}(T) X^{j} X^{k} \\
\overline{\boldsymbol{\Gamma}}_{\hat{\jmath}}^{(\mathrm{S})}= & -\left[\frac{1}{2}{ }^{F} R_{j 00 m}(T)+\frac{1}{3}^{F} R_{j l 0 m}(T) X^{l}\right] X^{m}, \\
\overline{\boldsymbol{\Gamma}}_{\hat{0}[\hat{l} \hat{m}]}^{(\mathrm{T})}= & \frac{1}{12}{ }^{F} R_{k[l m] j, 0}(T) X^{j} X^{k} \\
& +\frac{1}{3}\left[{ }^{F} R_{l m 0 k}(T)+{ }^{F} R_{k[l m] 0}(T)\right] X^{k}
\end{aligned}
$$

After defining $\boldsymbol{D}_{\hat{\mu}}=\boldsymbol{P}_{\hat{\mu}}-\hbar \boldsymbol{\Gamma}_{\hat{\mu}}$ and using the identity

$$
\gamma^{\hat{\mu}} \gamma^{\hat{\nu}} \gamma^{\hat{\rho}}=\eta^{\hat{\nu} \hat{\rho}} \gamma^{\hat{\mu}}-2 \gamma^{[\hat{\nu}} \eta^{\hat{\rho}] \hat{\mu}}-i \gamma^{5} \gamma^{\hat{\sigma}} \varepsilon^{\hat{\mu} \hat{\nu} \hat{\rho}} \hat{\sigma}
$$

where $\varepsilon^{\hat{\mu} \hat{\nu} \hat{\rho} \hat{\sigma}}$ is the Levi-Civita symbol with $\varepsilon^{\hat{0} \hat{1} \hat{2} \hat{3}}=1$, it follows that

$$
\begin{aligned}
\boldsymbol{D}_{\hat{\mu}} & =\boldsymbol{P}_{\hat{\mu}}-\hbar\left(\gamma^{5} \overline{\boldsymbol{\Gamma}}_{\hat{\mu}}^{(\mathrm{C})}-i \overline{\boldsymbol{\Gamma}}_{\hat{\mu}}^{(\mathrm{S})}\right), \\
\overline{\boldsymbol{\Gamma}}_{\hat{\mu}}^{(\mathrm{C})} & =\varepsilon_{\hat{\mu}}^{\hat{0} \hat{l} \hat{m}} \overline{\boldsymbol{\Gamma}}_{\hat{0}[\hat{l} \hat{m}]}^{(\mathrm{T})},
\end{aligned}
$$

where "S" gives the symmetric part of the spin connection under chiral symmetry and "C" implies chiral dependence by its coupling with $\gamma^{5}$.

Following standard definitions, the Pauli-Lubanski vector in a local Lorentz frame is

$$
\boldsymbol{W}^{\hat{\mu}}=-\frac{1}{4} \varepsilon_{\hat{\alpha} \hat{\beta} \hat{\gamma}}^{\hat{\mu}} \sigma^{\hat{\alpha} \hat{\beta}} \boldsymbol{D}^{\hat{\gamma}} \text {. }
$$

Using both (7) and the identity $\varepsilon_{\hat{\mu} \hat{\nu} \hat{\rho} \hat{\sigma}} \sigma^{\hat{\rho} \hat{\sigma}}=-2 i \gamma^{5} \sigma_{\hat{\mu} \hat{\nu}}$, its squared magnitude is

$$
\boldsymbol{W}^{\hat{\alpha}} \boldsymbol{W}_{\hat{\alpha}}=-\frac{3}{4} \boldsymbol{D}^{\hat{\alpha}} \boldsymbol{D}_{\hat{\alpha}}+\frac{i}{4} \sigma^{\hat{\alpha} \hat{\beta}}\left[\boldsymbol{D}_{\hat{\alpha}}, \boldsymbol{D}_{\hat{\beta}}\right]
$$


and leads to

$$
\begin{aligned}
\boldsymbol{W}^{\hat{\alpha}} \boldsymbol{W}_{\hat{\alpha}}= & -\frac{3}{4}\left[m_{0}^{2}+\frac{\hbar^{2}}{6}\left({ }^{F} R^{\hat{\alpha}}{ }_{\hat{\alpha}}\right)\right]+\frac{\hbar}{2}(\boldsymbol{\sigma} \cdot \boldsymbol{R}) \\
& -\frac{\hbar}{4} \sigma^{\hat{\alpha} \hat{\beta}} Q_{\hat{\alpha} \hat{\beta}}+\frac{3}{2} \hbar\left(\gamma^{5} \overline{\boldsymbol{\Gamma}}_{\hat{\alpha}}^{(\mathrm{C})}-i \overline{\boldsymbol{\Gamma}}_{\hat{\alpha}}^{(\mathrm{S})}\right) \boldsymbol{P}^{\hat{\alpha}} \\
& +\frac{3}{4} \hbar^{2} \boldsymbol{\nabla}^{\hat{\alpha}}\left(\overline{\boldsymbol{\Gamma}}_{\hat{\alpha}}^{(\mathrm{S})}+i \gamma^{5} \overline{\boldsymbol{\Gamma}}_{\hat{\alpha}}^{(\mathrm{C})}\right)
\end{aligned}
$$

where $m_{0}^{2}=\boldsymbol{P}^{\hat{\alpha}} \boldsymbol{P}_{\hat{\alpha}}$, the Casimir invariant for momentum, $\boldsymbol{\nabla}_{\hat{\mu}}$ is the gradient operator in curvilinear coordinates, and $Q_{\hat{\alpha} \hat{\beta}}$ is purely curvature-dependent. When ${ }^{F} R_{\mu \nu \alpha \beta}(T) \rightarrow 0$, (12) reduces to its flat space-time expression [6], where $\frac{\hbar}{2}(\boldsymbol{\sigma} \cdot \boldsymbol{R})$ is the non-inertial dipole interaction term to justify the given name for $\boldsymbol{R}$.

Extension for Noncommutative Geometry.-To ensure that (3) is truly symmetric requires assuming that the Riemann tensor is also symmetric in the middle two indices. Since this is generally not true, the symmetric properties of ${ }^{F} g_{\mu \nu}(X)$ follow directly from assuming the condition that $s^{2}={ }^{F} g_{\mu \nu}(X) X^{\mu} X^{\nu}$, where $X^{\mu}$ and $\mathrm{X}^{\mu}$ are ordinary c-numbers. This is an important observation given that the antisymmetric combination ${ }^{F} R_{\mu[\alpha \beta] \nu}(T)=\frac{1}{2}\left[{ }^{F} R_{\mu \alpha \beta \nu}(T)-{ }^{F} R_{\mu \beta \alpha \nu}(T)\right]$ leads to the appearance of terms like

$$
{ }^{F} R_{\mu[\alpha \beta] \nu}(T) X^{\mu} X^{\nu}=-\frac{1}{4}{ }^{F} R_{\alpha \beta \mu \nu}(T)\left[X^{\mu}, X^{\nu}\right] .
$$

Assuming that $X^{\mu}$ are ordinary c-numbers, it follows that (13) automatically vanishes. However, if $X^{\mu} \rightarrow X^{\mu}$, then (13) is nonzero from (1), which results in $s^{2} \rightarrow$ $\frac{1}{2}{ }^{F} g_{(\mu \nu)}(\boldsymbol{X})\left(\boldsymbol{X}^{\mu} \otimes \boldsymbol{X}^{\nu}+\boldsymbol{X}^{\nu} \otimes \boldsymbol{X}^{\mu}\right)+\frac{1}{2} F g_{[\mu \nu]}(\boldsymbol{X}) \boldsymbol{X}^{\mu} \wedge$ $\boldsymbol{X}^{\nu}$, where ${ }^{F} g_{[\mu \nu]}(\boldsymbol{X})$ is a Hermitian operator defined at the $k$-scale, and

$$
\begin{aligned}
{ }^{F} g_{[\mu j]}(\boldsymbol{X})= & \frac{i \hbar}{6}\left[\delta^{0}{ }_{\mu}{ }^{F} R_{0 j k l}(T)+\frac{1}{2} \delta^{i}{ }_{\mu}{ }^{F} R_{i j k l}(T)\right] \\
& \times \boldsymbol{J}^{k l}(\boldsymbol{X}) .
\end{aligned}
$$

This leads to an immediate extension of the spin connection, in the form

$$
\begin{aligned}
\overline{\boldsymbol{\Gamma}}_{\hat{\mu}}^{(\mathrm{S})} & \rightarrow \overline{\boldsymbol{\Gamma}}_{\hat{\mu}}^{(\mathrm{S})}+\frac{i \hbar}{12} F^{F} R_{l m 0 \mu, 0}(T) \boldsymbol{J}^{l m}, \\
\overline{\boldsymbol{\Gamma}}_{\hat{\mu}}^{(\mathrm{C})} & \rightarrow \overline{\boldsymbol{\Gamma}}_{\hat{\mu}}^{(\mathrm{C})}+\frac{i \hbar}{48} \varepsilon^{\hat{0} \hat{\jmath} \hat{k}}{ }_{\hat{\mu}}^{F} R_{l m j k, 0}(T) \boldsymbol{J}^{l m},
\end{aligned}
$$

which subsequently introduces non-trivial terms to (10).

Moffat's Nonsymmetric Gravity Theory.-It is reasonable to surmise that metric corrections due to noncommutative geometry, as proposed by (14), are very small. Nevertheless, by allowing for ${ }^{F} g_{[\mu \nu]}(\boldsymbol{X}) \neq 0$, it is clear that a generalization away from GR is still required to accommodate a nonsymmetric description of gravity. The theory put forward by Moffat [10, 11] proposes that both the metric tensor $g_{\mu \nu}$ and the connection $\Gamma^{\lambda}{ }_{\mu \nu}$ are nonsymmetric in their lower indices. Furthermore, the modified curvature tensor is described using the nonsymmetric connection

$$
W_{\mu \nu}^{\lambda}=\Gamma_{\mu \nu}^{\lambda}-\frac{2}{3} \delta_{\mu}^{\lambda} W_{\nu},
$$

where $W_{\mu}=W^{\lambda}{ }_{[\mu \lambda]}$ is a vector field expressed in terms of $g_{[\mu \nu]}$. The modified tensor components in the Fermi frame are then introduced into the orthonormal vierbeins $\left\{\bar{e}^{\mu}{ }_{\hat{\alpha}}(X)\right\}$ and inverse vierbeins $\left\{\bar{e}^{\hat{\alpha}}{ }_{\mu}(X)\right\}[8]$. Moffat's theory is particularly useful in the linear approximation about the Minkowski metric [11], since the symmetric and antisymmetric field equations decouple to first order. Given that ${ }^{F} g_{\mu \nu}(X)$ according to (3) is expressible precisely as a linearized expansion about $\eta_{\mu \nu}$, this particular form of Moffat's nonsymmetric gravity theory is especially useful in this context.

Noncommutative Geometry in Muon Decay.-For this paper, the decay process occurs for a muon in circular orbit around a microscopic Schwarzschild black hole 8] of mass $M=3 \times 10^{-12} \mathrm{~cm}$ with (classical) orbital radius $r_{0} \sim 6 M$, where $X^{j}$ represents spatial quantum fluctuations about $r_{0}$. The matrix element [6, 8] for the muon decay reaction $\mu^{-} \rightarrow e^{-}+\bar{\nu}_{e}+\nu_{\mu}$ is

$$
|\mathcal{M}|^{2}=\frac{G_{\mathrm{F}}^{2}}{2} L_{\hat{\mu} \hat{\nu}}^{(\mu)} M_{(e)}^{\hat{\mu} \hat{\nu}},
$$

where

$L_{(\mu)}^{\hat{\mu} \hat{\nu}}=\operatorname{Tr}\left[\boldsymbol{p}_{\nu_{\mu}} \gamma^{\hat{\mu}}\left(\boldsymbol{D}_{\mu}+m_{\mu} \gamma^{5} \boldsymbol{h}_{\mu}\right) \gamma^{\hat{\nu}}\left(1-\gamma^{5}\right)\right]$

$M_{(e)}^{\hat{\mu} \hat{\nu}}=\operatorname{Tr}\left[\left(\boldsymbol{D}_{e}+m_{e} \gamma^{5} \boldsymbol{h}_{e}\right) \gamma^{\hat{\mu}} \boldsymbol{p}_{\nu_{e}} \gamma^{\hat{\nu}}\left(1-\gamma^{5}\right)\right]$,

and $\boldsymbol{n}^{\hat{\mu}}$ is the polarization vector for the charged lepton. From the orthogonality condition $\boldsymbol{n}^{\hat{\mu}} \boldsymbol{D}_{\hat{\mu}}=0$, it can be shown [8] that $\boldsymbol{h}=\left[\boldsymbol{h}^{(\mathrm{S}, \mathrm{Re})}+i \boldsymbol{h}^{(\mathrm{S}, \mathrm{Im})}\right]+$ $\gamma^{5}\left[\boldsymbol{h}^{(\mathrm{C}, \mathrm{Re})}+i \boldsymbol{h}^{(\mathrm{C}, \mathrm{Im})}\right]$, eventually leading to

$$
|\mathcal{M}|^{2}=32 G_{\mathrm{F}}^{2}\left(\boldsymbol{P}_{\nu_{e}}^{\hat{\alpha}} \overline{\boldsymbol{D}}_{\hat{\alpha}}^{\mu}\right)\left(\boldsymbol{P}_{\nu_{\mu}}^{\hat{\beta}} \overline{\boldsymbol{D}}_{\hat{\beta}}^{e}\right)
$$

where $\overline{\boldsymbol{D}}_{\hat{\alpha}}=\boldsymbol{P}_{\hat{\alpha}}-\hbar\left(\overline{\boldsymbol{\Gamma}}_{\hat{\alpha}}^{(\mathrm{C})}-i \overline{\boldsymbol{\Gamma}}_{\hat{\alpha}}^{(\mathrm{S})}\right)+m_{0}\left(\boldsymbol{n}_{\hat{\alpha}}^{(\mathrm{C})}-\boldsymbol{n}_{\hat{\alpha}}^{(\mathrm{S})}\right)$. Both the gravitational and $\boldsymbol{R}$-dependent contributions to the muon decay rate are additive corrections to $\Gamma_{0} \approx$ $G_{\mathrm{F}}^{2} m_{\mu}^{5} /\left(192 \pi^{3}\right) \approx 2.965 \times 10^{-16} \mathrm{MeV}[8]$. As well, $|\mathcal{M}|^{2}$ formally contains new terms due to noncommutative geometry when accounting for (15) and the additional curvature terms due to Moffat's theory.

For this paper, $\boldsymbol{J}^{\mu \nu}$ is defined according to two possible choices [13]:

$$
\begin{aligned}
\boldsymbol{J}^{\mu \nu}(\boldsymbol{X}) & =i C_{(0)}^{\mu \nu}, & & (\text { Case 1) } \\
& =\frac{i}{r_{0}} C_{(1) \gamma}^{\mu \nu} \boldsymbol{X}^{\gamma}, & & (\text { Case 2) }
\end{aligned}
$$

where $C_{(0)}^{\mu \nu}$ and $C_{(1) \gamma}^{\mu \nu}$ are dimensionless real-valued structure constants chosen with $C_{(0)}^{i j}=C_{(1)}^{i j}=+1$ for $i j=\{12,23,31\}$, and all others equal to zero. When applied to Moffat's nonsymmetric theory [11], it is shown 


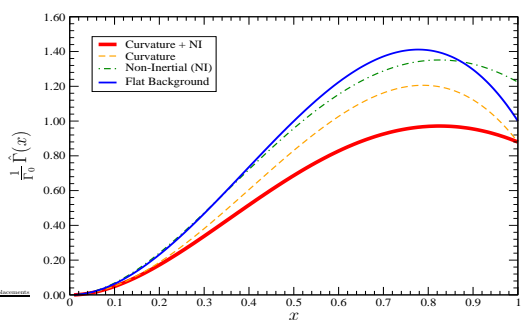

(a) $\quad M=3 \times 10^{-12} \mathrm{~cm}\left(r_{0} \sim 6 M\right)$

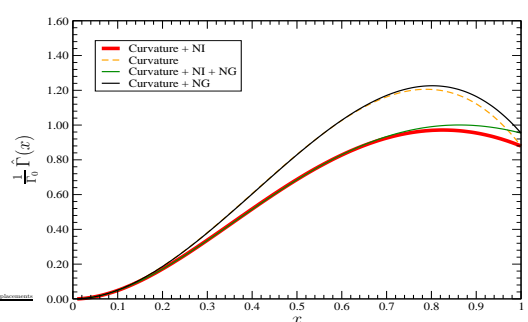

(b) $\sqrt{\hbar}=5 \times 10^{-9} \mathrm{~cm}($ Case 1$)$

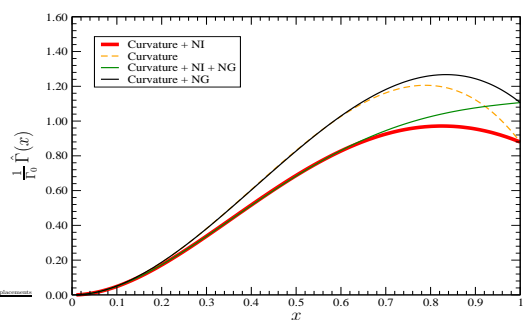

(c) $\sqrt{\hbar}=2 \times 10^{-8} \mathrm{~cm}($ Case 2$)$

FIG. 1: Michel spectrum for muon decay while in circular orbit around a microscopic Schwarzschild black hole of mass $M=3 \times 10^{-12} \mathrm{~cm}$ and orbital radius $r_{0} \sim 6 M$. Fig. 1(a) shows the various profiles available, including both curvature and $\boldsymbol{R}$-dependent non-inertial contributions (NI) [8], minus the predictions due to noncommutative geometry (NG). Fig. 1(b) shows the contributions due to noncommutative geometry for Case 1, given by (20a), while Fig. 1(c) shows the corresponding description for Case 2, given by (20b).

that the components for $W_{\mu}$ (as c-numbers) in the Fermi frame for Case 1 are

$$
{ }^{F} W_{\mu}(X)=\frac{\hbar}{3} F_{k l 0 \mu, 0}(T) C_{(0)}^{k l},
$$

while the corresponding components for Case 2 are

$$
\begin{aligned}
{ }^{F} W_{0}(X)= & \frac{\hbar}{3 r_{0}}{ }^{F} R_{k l m 0}(T) C_{(1)}^{k l m}, \\
{ }^{F} W_{j}(X)= & \frac{\hbar}{3 r_{0}}\left[{ }^{F} R_{k l 0 j, 0}(T) C_{(1) m}^{k l} X^{m}\right. \\
& \left.+\frac{1}{2}{ }^{F} R_{k l m j}(T) C_{(1)}^{k l m}\right] .
\end{aligned}
$$

Analysis and Conclusion.-All computations in this paper are performed in the muon's local rest frame. Figure 1 shows the Michel spectrum $\hat{\Gamma}(x)=\Gamma / x$ in units of $\Gamma_{0}$ as a function of the outgoing electron energy fraction $x$. It is clear from Fig. 1(a) in the absence of noncommutative geometry that space-time curvature reduces the decay rate [8]. Figs. 1(b) and 1(c) reveal the presence of $k$ due to Cases 1 and 2 , respectively, where the change of profile occurs for $x \gtrsim 0.65$. It is interesting to note that this contribution serves to counteract the emergent curvature-induced stabilization at the muon's Compton wavelength scale for the adopted sign convention of the structure constants. As well, it is surprising to note that a signature appears only for terms quadratic in curvature but linear in $k$, as all such linear curvature terms identically vanish when time-averaged over an orbital cycle. The reasons for its occurrence are not known.

Identification of a discernable signal requires $\sqrt{\hbar} \sim$ $10^{-9} \mathrm{~cm}$ for Case 1, about an order of magnitude smaller than for Case 2. While this length scale for $\sqrt{\hbar}$ is within the realm of possibility and is strictly speaking an unknown quantity, its value is at least ten orders of magnitude larger than the theoretical upper bound of $\sqrt{\hbar} \sim 10^{-18} \mathrm{~cm}[14]$ predicted in the context of noncommutative QED in flat space-time. Since this treatment of noncommutative geometry requires a curved space-time background to manifest its existence, it is unclear how the empirically-motivated choices for $\sqrt{\hbar}$ in Figs. 1(b) and 1(c) relate with the theoretical motivations given elsewhere. Irrespective of whether the Large Hadron Collider (LHC) can produce Schwarzschild black holes without invoking large extra spatial dimensions, it is also unclear whether direct observational evidence for noncommutative geometry can be gained by this method. Nonetheless, this approach at least provides an interesting avenue for further exploration.

* Electronic address: dinesh.singh@uregina.ca

$\dagger$ Electronic address: nader.mobed@uregina.ca

¥ Electronic address: pierre-philippe.ouimet@uregina.ca

[1] J. Polchinski, String Theory (Cambridge University Press 1998); M.B. Green, J.H. Schwarz, and E. Witten, Superstring Theory (Cambridge University Press 1987).

[2] A. Ashtekar, Phys. Rev. D 36, 1587 (1987); Phys. Rev. Lett. 57, 2244 (1986); C. Rovelli, Quantum Gravity (Cambridge University Press, 2004).

[3] A. Connes, Commun. Math. Phys. 192, 155 (1996); P. Aschieri, M. Dimitrijevic, F. Meyer and J. Wess, Class. Quant. Grav. 23, 1883 (2006).

[4] H.S. Snyder, Phys. Rev. 71, 38 (1947).

[5] P. Nicolini, arXiv:0807.1939v1 [hep-th].

[6] D. Singh and N. Mobed, Class. Quantum Grav. 24, 2453 (2007).

[7] D. Singh, Phys. Rev. D 71, 105003 (2005); D. Singh, N. Mobed, and G. Papini, J. Phys. A 37, 8329 (2004).

[8] D. Singh and N. Mobed, Phys. Rev. D 79, 024026 (2009).

[9] K. Rosquist, Class. Quantum Grav. 23, 3111 (2006).

[10] J.W. Moffat, Phys. Rev. D 19, 3554 (1979).

[11] J.W. Moffat, Phys. Lett. B355, 447 (1995).

[12] C.W. Misner, K.S. Thorne, and J.A. Wheeler, Gravitation (W.H. Freeman and Company 1973).

[13] J. Madore et al., Eur. Phys. J. C16, 161 (2000).

[14] M. Chaichian, M.M. Sheikh-Jabbari, and A. Tureanu, Phys. Rev. Lett. 86, 2716 (2001). 